\title{
Children and adolescents with headache: what do they need?
}

\author{
David Kernick · Deborah Reinhold
}

Received: 14 March 2008 / Accepted: 3 May 2008/Published online: 10 June 2008

(c) Springer-Verlag 2008

\begin{abstract}
Headache is the most frequent neurological symptom and common manifestation of pain in children. The needs of this group are poorly understood. The aim of this study is to quantify the extent of unmet need in a primary care paediatric population. A patient questionnaire survey of 2,425 children between the ages of 8 and 17 and an uncontrolled intervention study was undertaken in a large general practice in England. Headache impact was measured before and 4 months after a headache clinic intervention using the paediatric migraine disability assessment score. A total number of 74 (3\%) children accepted an invitation for a headache assessment. However, only $49(2 \%)$ attended for consultation of whom $84 \%$ were judged to have migraine. A total number of $43(58 \%)$ had been seen before for a headache by their general practitioner. The median impact score was 17 days headache impact in a 3-month period. An intervention by a general practitioner with an interest in headache significantly reduced the headache impact score. There is a significant need amongst children with headache that can be addressed by interventions well within the capacity of general practitioners. Further studies are needed to explore why so few children with headache present to primary care and when they do, why their needs are not adequately addressed.
\end{abstract}

Keywords Headache - Children · Primary care

D. Kernick $(\bowtie) \cdot$ D. Reinhold

Research Unit, St Thomas Medical Group, St Thomas Health

Centre, Cowick Street, Exeter EX4 1HJ, UK

e-mail: su1838@eclipse.co.uk

\section{Background}

Despite a substantial burden, the needs of adults with headache are often unmet $[1,2]$. When they do seek help, the diagnosis is often incorrect and the condition poorly managed [3]. In the paediatric population, despite being the most frequent neurological symptom and common manifestation of pain, the impact of headache on the quality of life and the health needs of these patients are poorly understood. Barriers to care may be more prominent as children are less able to articulate their problems and seek help.

Headache can be primary (migraine and tension-type in this population) and secondary where an underlying pathology can be identified. In the paediatric population, migraine is the most common type of headache with a peak incidence at the age of 15 and 10 years in females and males, respectively [4]. A large UK school clinical study reported an annual prevalence rate of $10.6 \%$ for migraine and $0.9 \%$ for tension type headache [5]. The prevalence of patient self-reported headache is higher. Between 20 and $30 \%$ of children report headaches at least weekly and $6 \%$ have headaches several times a week or daily [6-8].

Headache has an impact on a child's life in a number of ways including school absences, reduction in performance, decreased home and family interactions and decreased socialisation with peers [9]. Patients attending a specialist paediatric headache clinic have similar impact on the quality of their lives compared to children suffering from cancer and arthritis [10]. Headache during childhood has a high risk of persisting into adulthood [11] with associated physical and psychiatric morbidity later in life [12].

The aim of this study was to explore the medical needs of children and adolescents with headache in a primary care setting. 


\section{Method}

Setting

The study was undertaken at a large general practice in Exeter (UK). The practice population is of average socioeconomic status lying on the 48th centile of the England and Wales index of multiple deprivations (IMD). The IMD contains seven domains of deprivation that are available at a small area level and is a proxy for socio-economic status [13].

\section{Target population}

All registered children of the practice between the ages of 8 and 17 were invited to participate. General practitioners (GPs) were given the option to exclude children who they thought unsuitable without reason. For example, those with ongoing serious illness where an approach would be inappropriate.

\section{Method}

We sent a letter to children and their parents asking them if they had headache that impacted upon the quality of their life and if so, whether they wished to see a general practitioner with a special interest in the area for assessment and advise. Ethics approval was obtained from the North and East Devon Research Ethics Committee.

Children who accepted were asked to fill in a postal questionnaire prior to their clinic appointment that recorded frequency and impact of their headache using the paediatric migraine disability assessment score (PedMIDAS). This is a validated instrument that measures the impact of headache related quality of life in terms of days in the previous 3-month period that headache had impacted totally or partially on school or home life [14]. This questionnaire was repeated by post 4 months after the initial clinic appointment with one written reminder for non-responders.

The needs of patients were assessed by two GPs who took part in the intervention. Both had a special interest in headache and had provided an intermediate care headache clinic for the previous 5 years [15] (http://www.headache. exeter.nhs.uk). Initial appointments were for 20-min with $10 \mathrm{~min}$ follow-ups as required. As the International Headache Society criteria for the diagnosis of migraine in children has a low sensitivity [16], a pragmatic approach was taken to diagnosis based on our clinical experience.

For the headache group completing the initial questionnaire, previous primary care consultations were analysed and compared with a control group matched for age and sex who had no previous headache history.
Statistics

The main variable of interest, the PedMIDAS was not normally distributed and distributions are quoted in medians and upper and lower quartiles. Changes in PedMIDAS before and after clinic intervention were measured for significance using the Mann Whitney test, where normally distributed variables are quoted using means and standard deviations.

\section{Results}

Patient response

Exactly 2,461 children were identified between the ages of 8 and 17. Individual general practitioners requested that 36 patients were not contacted and 2,425 invitations were issued. A total number of 74 (3.0\%) children took up the offer of a headache assessment and completed an initial questionnaire. Their average age was $12.5( \pm 2.7)$. A total number of $16(23 \%)$ had headache six or more times in a month and 13 (17\%) had headache for more than 15 days in a month. The median PedMIDAS score was 17.5 (33.0, $17.5,10.0)$. A total number of 3.5 days of school were totally lost during this period.

Of those expressing a wish to be assessed, only 49 $(66 \%)$ actually attended for a consultation. There was no difference in the age or IMD of the groups that did and did not attend but the non-attendees had a higher impact score. There was a significant difference between those that did attend the clinic appointment who had a lower median PedMIDAS score of $14.5(31.0,14.5,10.0)$ compared with those who did not attend the clinic whose median score was 24 (40.0, 24.0, 10.0) $(P<0.05)$ (see Table 1).

Previous health seeking behaviour

A total number of $43(58 \%)$ patients who accepted an invitation had been seen previously for their headache problem with an average number of headache consultations of 1.7. The median number of non-headache consultations in the previous 3 years was $7.8(10.0,7.8,3.0)$ compared with the control group of $5.5(8.0,5.5,2.0) .(P>0.05$, non-significant difference).

\section{Clinical process}

Diagnostic categories of patients that attended for review were migraine or probable migraine $41(84 \%)$, mixed migraine and tension-type $2(4 \%)$, tension-type $5(10 \%)$. In one case a diagnosis was not made and one case was complicated by medication overuse. The average number of 
Table 1 Characteristics of respondents

\begin{tabular}{|c|c|c|c|c|}
\hline & $\begin{array}{l}\text { Respondents } \\
(N=74)\end{array}$ & $\begin{array}{l}\text { Did not attend } \\
\text { clinic appointment } \\
(N=25)\end{array}$ & $\begin{array}{l}\text { Attended } \\
\text { clinic appointment } \\
(N=49)\end{array}$ & $\begin{array}{l}\text { Seen in clinic and } \\
\text { returned final } \\
\text { questionnaire }(N=32)\end{array}$ \\
\hline Age (years) & $12.5 \pm 2.7$ & $12 \pm 2.6$ & $12.7 \pm 2.7$ & $13 \pm 2.9$ \\
\hline Number of females $(\%)$ & $39(53)$ & $12(48)$ & $27(55)$ & $18(56)$ \\
\hline \multicolumn{5}{|l|}{ Headache frequency } \\
\hline Less than one per month & $5(6 \%)$ & $2(8 \%)$ & $3(6 \%)$ & $9(28 \%)$ \\
\hline $1-6$ per month & $40(54 \%)$ & $15(60 \%)$ & $25(51 \%)$ & $18(56 \%)$ \\
\hline More than six per month & $16(23 \%)$ & $4(16 \%)$ & $12(25 \%)$ & $3(9 \%)$ \\
\hline On more than 15 days a month & $13(17 \%)$ & $4(16 \%)$ & $9(18 \%)$ & $2(6 \%)$ \\
\hline $\begin{array}{l}\text { PedMIDAS (days in previous } 3 \text { month period } \\
\text { totally or partially affected by headache) }\end{array}$ & $17.5(33,17.5,10.0)$ & $24.0(40.0,24.0,10.0)$ & $14.5(31.0,14.5,10.0)$ & $6.0(10.5,6.0,3.0)$ \\
\hline
\end{tabular}

follow-up appointments for each patient was 0.4. All patients received dietary and lifestyle advice. This included advice on fluid and food intake, the identification of stress at home and school and exploration of sleeping patterns. Interventions are shown in Table 2. Drugs were prescribed according to guidelines in the paediatric British National Formulary.

The PedMIDAS score of the $32(65 \%)$ of clinic attendees that returned a questionnaire at 4 months following their initial visit fell to 6.0 days $(10.5,6.0,3.0),(P=0.005)$. The number of sufferers with headache for more than 6 days in a month fell from 25 to $9 \%$ and for more than 15 days from 18 to $6 \%$. On an intention to treat analysis the PedMIDAS fell to 8.0 days, $(17.5,8.0,4.0),(P=0.01)$.

\section{Discussion}

\section{Summary of main findings}

We identified $3 \%$ of a primary care practice population aged between 8 and 17 years who wanted improvement in their headache management. This population demonstrated a high headache impact with a median of 17 days affected in a 3 month period including loss of 3.5 school days. Exactly $17 \%$ of this group had headache for more than 15 days of each month.

Table 2 Headache interventions delivered during the study. All patients received dietary and lifestyle advice

\begin{tabular}{lc}
\hline Intervention & Number of patients \\
\hline Dietary/lifestyle advice only & $3(6 \%)$ \\
Paracetamol/ibuprofen with/without & $31(63 \%)$ \\
$\quad$ domperidone & \\
Pizotifen & $16(33 \%)$ \\
Propranolol & $3(6 \%)$ \\
Amitriptyline & $3(6 \%)$ \\
Sumatriptan nasal spray & $9(18 \%)$ \\
\hline
\end{tabular}

Thirty-four percent of those that accepted our initial invitation for review did not attend for consultation, despite a higher headache impact than those that did attend. This was not due to socio-economic differences that may have been reflected in health seeking behaviour as both groups had the same socio-economic scores. Possible reasons were: the headache problem had resolved (unlikely as they were asked to make an appointment as soon as the 3-month headache monitoring period had been completed), a reflection of parental behaviour (the parents of children with migraine are likely to suffer themselves and the majority of the adult sufferers do not seek help), under-recognition of the problem (young children are often unable to articulate their headache problem which can present in other ways).

We were able to demonstrate a reduction in headache frequency and impact using simple interventions well within the remit of general practitioners. Although 58\% of children had been seen previously for their headache by their GP, their headache impact was still substantial.

Study strengths and limitations

This is the first practice-based study of the impact of headache in children and was undertaken in a practice that reflected the socio-economic profile of the UK. We can make no claim about the effectiveness of our clinical intervention as it was uncontrolled and may reflect a regression to the mean. However, when evidence-based headache interventions are delivered to sufferers, the expectation is that some will receive benefit. We also avoided any attempt at diagnosing and managing psycho-social factors. The relationship between headache and psycho-social factors is complex, contested and beyond the scope of this study.

Comparison with existing literature

The cohort of children we have identified sits between two other studies that have reported using the PedMIDAS as an 
impact assessment. Of those most severely affected, patients attending a US paediatric headache centre report an average PedMIDAS of 44 days [10]. A recent Turkish school survey of 7,700 children reported a migraine prevalence of $9.7 \%$, with average PedMIDAS scores of 9.9 days in boys and 11.5 days in girls [17].

The reluctance to consult for headache that we report has been confirmed in other studies. These suggest that $11-52 \%$ of children with headache do not seek medical help [18, 19].

Although differences were not significant, the higher nonheadache consultation rate in our headache group compared with matched controls may reflect other studies that suggest that headache sufferers experience more somatic complaints, lower general well-being and more physiologic anxiety than headache free controls [20-22]. This highlights the importance of broader approaches to headache management such as behavioural and psychological interventions in addition to pharmacological therapies in patients with headache.

Implications for future research

This study has highlighted a number of areas for further research. We need to explore why despite such high impact, so few children with headache present to primary care, even when invited to do so. Research is also needed to explore the barriers that prevent GPs from delivering adequate care.

\section{Conclusion}

In conclusion, although our study has a number of weaknesses, we have demonstrated a significant unmet need in the paediatric headache population that can be addressed by interventions well within the capacity of general practitioners. The headache impact we have identified is likely to be an under estimate as the barriers to headache care that have been identified in an adult population are likely to be more prominent in the paediatric population who are less able to articulate their problems and seek help.

Acknowledgments We thank Professor John Campbell for his helpful comments on an earlier draft. This work was undertaken with a grant from the Migraine Trust. St Thomas Medical Group is a Research and Development Practice that receives funding from the NHS R\&D executive.

Conflict of interest None.

\section{References}

1. Harpole L, Samsa G, Matchar D et al (2005) Burden of illness and satisfaction with care amongst patients with headache seen at their primary care setting. Headache 45(8):1048-1055
2. Dowson A, Jagger S (1999) The UK migraine patient survey: quality of life and treatment. Curr Med Res Opin 15:241-253

3. Lipton RB, Scher A, Steiner T, Bigal M, Kolodner K, Liberman J, Stewart W (2003) Patterns of health care utilisation for migraine in England and in the US. Neurology 60(3):441-448

4. Stewart W, Linet M, Celentano D, Van Natta M, Ziegler D (1991) Age and sex specific incidence rates of migraine with and without visual aura. Am J Epidemiol 134:1111-1120

5. Abu-Arefeh I, Russell G (1994) Prevalence of headache and migraine in school children. BMJ 309:765-769

6. Bandell-Hoekstra I, Abu-Saad H, Passchier J, Frederiks C, Feron F, Knipschild P (2001) Prevalence and characteristics of headache in Dutch schoolchildren. Eur J Pain 5:145-153

7. Kristjanstottir G, Wahlberg V (1993) Sociodemographic differences in the prevalence of self reported headache in Icelandic schoolchildren. Headache 33:376-380

8. Carlsson J (1996) Prevalence of headache in schoolchildren: relation to family and school factors. Acta Paediatr 85:692-696

9. Karwautz A, Wober C, Lang T, Bock A, Wagner-Ennsgraber C, Vesely C, Kienbacher C, Wober-Bingol C (1999) Psychosocial factors in children and adolescents with migraine and tension type headache: a controlled study and review of the literature. Cephalalgia 19(1):32-43

10. Scott W, Powers SW, Patton SR, Hommel KA, Hershey A (2003) Quality of life in childhood migraines: clinical impact and comparison to other chronic illnesses. Paediatrics 112(1):1-5

11. Guidetti V, Galli F (1998) Evolution of headache in childhood and adolescents: an 8-year follow up. Cephalalgia 18:449-454

12. Fearon P, Hotopf M (2001) Relation between headache in childhood and physical and psychiatric symptoms in adulthood: national birth cohort study. BMJ 322:1-6

13. The English Indices of Deprivation 2004. Office of the Deputy Prime Minister, London (http://www.communities.gov.uk)

14. Hershey A, Powers S, Vockell A, Lecates S, Kabbouche MA, Maynard M (2001) PedMIDAS: development of a questionnaire to assess disability of migraines in children. Neurology 57:2034 2039

15. Kernick D (2005) A descriptive study of an intermediate care headache clinic delivered by general practitioners with a special interest. Headache Care 2(2):101-104

16. Lima M, Padula N, Santos L, Oliveira L, Ajapejev S, Padovani C (2005) Critical analysis of the International classification of headache disorders diagnostic criteria (ICHDI-1988) and (ICHDII-2004) for migraine in children and adolescents. Cephalalgia 25:1042-1047

17. Akyol A, Kiylioglu N, Aydin I, Erturk A, Kaya E, Telli E, Akyıldız U (2007) Epidemiology and clinical characteristics of migraine among school children in the Menderes region. Cephalalgia 27:781-787

18. Mortimer M, Kay J, Jarron A (1992) Childhood migraine in general practice: clinical features and characteristics. Cephalalgia 12:238-43

19. Metsahonkala L, Sillanpää M, Tuominen J (1996) Use of headache services in childhood migraine. Headache 36:423-428

20. Roth-Isigkeit A, Thyen U, Stoven H, Schwarzenberger J, Schmucker P (2005) Pain among children and adolescents: restrictions in daily living and triggering factors. Paediatrics 115(4): 1118

21. Carlsson J, Larsson B, Mark A (1996) Psychosocial functioning in schoolchildren with recurrent headaches. Headache 36(2):7782

22. Bandell-Hoekstra I, Abu-Saad HH, Passchier J, Knipschild P (2000) Recurrent headache, coping, and quality of life in children: a review. Headache 40:357-370 